Seed Science Research

Volume 27 , Issue 3

September 2017 , pp. 243-250

\title{
Using automated sanding to homogeneously break seed dormancy in black locust (Robinia pseudoacacia L., Fabaceae)
}

Xavier P. Bouteiller (a1), Annabel J. Porté (a1), Stéphanie Mariette (a1) and Arnaud Monty (a2)

https://doi.org/10.1017/S0960258517000150

Published online: 05 June 2017

\begin{abstract}
Physical dormancy of Robinia pseudoacacia seeds makes it a challenge for scientists and forest managers to obtain a homogeneous germination for larger seed samples. Water imbibition of the seeds can be achieved through manual piercing of the seed coat, but this method remains time consuming and heterogeneous. We tested several ecologically friendly methods to break seed dormancy, including manual pin puncture, water soaking, oven dry-heating (two temperatures) and sanding. Sanding was performed using an automatic grinder to control shaking duration (three durations) and get a homogeneous scraping of the coat. All methods, except dry-heating, resulted in successful dormancy breaking; water soaking was the least efficient method, attaining $57 \%$ germination. Sanding proved to be as efficient as puncturing $(97 \%)$ but long duration sanding (10 or $15 \mathrm{~min}$ ) could damage cotyledons, which would impede further development of the plant. Short-time sanding $(5 \mathrm{~min}$ ) proved to be the best method to reach high total germination and healthy (undamaged cotyledon) seedlings, and was successfully applied to 500 seeds. The reference puncture method and the automatic sanding were also tested on seeds of nine Fabaceae species and proved to be efficient for some species. Automated sanding can thus be used as a standard to break physical dormancy of black locust or other Fabaceae seeds to allow further comparative studies of plant populations or genotypes.
\end{abstract}

